\title{
Política integral fronteriza y derecho migratorio: Una mirada desde las vivencias de migrantes venezolanos ${ }^{1}$
}

\author{
Integral border policy and migration law: \\ A view from the experiences of Venezuelan migrants
}

JUlio CÉSAR CARVAJAl RodRígueZ ${ }^{2}$

JosÉ DE JESÚS NÚÑEZ RODRÍGUEZ

PAUlo CÉSAR Lugo RINCÓN ${ }^{4}$

$0^{\circ}$ IUS Comitiãlis / Año 4, Número 7 / enero - junio 2021 / pp. 119-139 / ISSN: 2594-1356

Recepción: 26 de mayo de 2020 / Aceptación: 27 de septiembre de 2020

Resumen: Los fenómenos migratorios convocan con urgencia a repensar la forma en que los países los afrontan. Al respecto, se ha vuelto preponderante trabajar en una reingeniería de las políticas públicas existentes. El presente artículo es el resultado de la investigación desarrollada en la zona de frontera colombo venezolana, en la ciudad de Cúcuta, durante los años 2018-2020, y está orientada al análisis de las diferentes vivencias y percepciones de tres migrantes venezolanos en territorio colombiano, de dos entidades encargadas de su atención (ACNUR), Migración Colombia y dos expertos en tema migratorios. A partir de los hallazgos encontrados se proponen aportes que pueden ser importantes a la hora de pensar en la construcción de una política pública integral sobre la migración.

Palabras clave: Migración, política pública integral, derecho migratorio, migrante.

Abstract: Migration phenomena urgently call for a rething of the way countries deal with them. In this regard, it has become predominant to work on a reengineering of existing public policies. This article is the result of research carried out in the Area of Venezuelan Colombo border, in the city of Cúcuta, during the years 2018-2020, and is oriented to the analysis of the different experiences and perceptions of three Venezuelan migrants in Colombian territory, two entities responsible for their attention (UNHCR), Migration Colombia and two experts in migration. From the findings found, contributions are proposed that may be important when thinking about the construction of a comprehensive public policy on migration.

Key words: Migration, integral public policy, migration law, migrant.

${ }^{1}$ En la elaboración de este artículo los autores agradecen la colaboración de Víctor Roseliano Díaz Quero, Centro de Investigación “Georgina Calderón” de la Universidad Pedagógica Experimental Libertador - Caracas, Venezuela.

http://orcid.org/0000-0003-4517-8817. / Correo electrónico: departamentodeeducacion@cúcuta.udes.edu.co ${ }^{2}$ Universidad de Santander UDES, Facultad de Ciencias de la Educación, Departamento de Educación; Cúcuta, Departamento Norte de Santander Colombia.

http://orcid.org/0000-0002-4120-0215. / Correo electrónico: jo.nunez@mail.udes.edu.co

${ }^{3}$ Universidad de Santander, Facultad de Ciencias Económicas, Administrativas y Contables, Grupo de Investigación CIEMPIÉS, Bucaramanga, Colombia. Ciudad, Estado y País: Cúcuta, Departamento Norte de Santander, Colombia.

https://orcid.org/0000-0002-1571-9080. / Correo electrónico: paulolugo@hotmail.com

${ }^{4}$ Facultad de Ciencias Políticas y Relaciones Internacionales de la UANL., Monterrey, Nuevo León, México. 


\section{INTRODUCCIÓN}

Los efectos de los altos volúmenes de los flujos migratorios de venezolanos en la zona fronteriza de Cúcuta, Colombia, en la línea limítrofe con la República de Venezuela, ha generado un impacto que evidencia los diferentes problemas sociales y económicos que se han venido agudizando, ante la incapacidad de los entes gubernamentales y el agotamiento humano y presupuestal de las entidades internacionales generado por el desbordamiento del desplazamiento de personas.

A diario los medios de comunicación y las redes sociales registran la problemática migratoria y lo que implica un hacinamiento en donde los más afectados son los grupos etarios como los niños, ancianos, mujeres embarazadas y la comunidad indígena Yukpa; migrantes en situación precaria son un grupo de personas vulnerables y heterogéneas (Féte, Aho, Benoit, Cloos y Ridde, 2019).

Este fenómeno, no solo ha afectado a la población vulnerable sino que se ha generalizado a toda la población migrante, lo que ha desbordado la capacidad de atención humanitaria de los gobiernos locales, colocándonos frente al reto de cómo asumir la problemática; tarea ejercitada en esta investigación a partir de trato directo con los diferentes actores implicados en el proceso, buscando desde un enfoque metodológico fenomenológico y hermenéutico, una sistematización de las categorías emergentes que puedan dar algunas recomendaciones a los gobiernos del hemisferio desde una nueva cosmovisión de la migración, lograr construir una política pública integral y efectiva al igual que fortalecer o actualizar la normatividad existente en temas de derecho migratorio. Ante el incremento migratorio, muchos estados se ven obligados a abordar constantes reajustes en sus políticas públicas, aceptando ciertas recomendaciones del derecho internacional (Guillén de Romero, Menéndez y Moreira, 2019). En esta misma dirección Mazuera, Albornoz, Cuberos, Vivas y Morffe (2020), plantean que la migración masiva venezolana ha provocado una crisis regional, ya que la región no está acostumbrada a recibir este tipo de inmigrantes, especialmente en las condiciones en las que huyen de Venezuela y luego llegan a las sociedades con las debilidades estructurales comunes en los países en desarrollo, lo que los ha llevado a asumir responsabilidades y compromisos, comenzando por reformular la legislación y las políticas migratorias, que han quedado obsoletas porque en su mayoría consideraban una perspectiva de seguridad y control.

Conocer esa problemática es pertinente para fortalecer las políticas incipientes que se han elaborado como política pública en el Consejo Nacional de Política Económica y Social (CONPES), creado por la Ley 19 de 1958; organismo que representa la máxima autoridad nacional de planeación y se desempeña como una entidad asesora del Gobierno Nacional en todos los aspectos relacionados con el desarrollo económico y social de Colombia. 
El presente artículo recoge las categorías emergentes de las diferentes vivencias y percepciones de los migrantes; entidades encargadas de su atención y de connotados expertos en temas migratorios y política pública, con el propósito de identificar las categorías más importantes que puedan ser un valor agregado a la construcción de una política pública integral a la luz del derecho migratorio, no solo para el estado colombiano, sino para otros países de la región.

\section{EL ABORDAJE METODOLÓGICO}

La investigación se realizó desde el paradigma pos-positivista-cualitativo, es decir el fenómeno estudiado por sus complejidades psicológicas, biológicas, socioculturales y propias de los sujetos migrantes, se mueven en ambientes históricos y contextos socioculturales de múltiples determinaciones, no sujetos entonces a enfoques causa-efecto propios del paradigma cuantitativo; para Martínez (2009, p. 168) "en los seres vivos, y sobre todo en los seres humanos, se dan estructuras de un altísimo nivel de complejidad y estos sistemas constituyen un todo físico-químico-biológico-cultural y espiritual".

Se empleó un muestreo intencional, al seleccionar una muestra de tres migrantes, dos entidades encargadas de la atención al migrante y dos expertos en el tema (tabla 1), realizándoles una entrevista a profundidad para develar las vivencias y percepciones de los sujetos objeto de la investigación. El desarrollo operativo de la investigación se apoyó en el método Introspectivo o Fenomenológico Interpretativo, orientado a develar e interpretar las diferentes categorías emergentes del estudio.

En la selección de los Informantes Clave (IC), los actores sociales, en este caso los migrantes que son de interés para la investigación, fue a través de criterios de selección. Rojas (2010), establece que la complejidad, heterogeneidad y los diferentes roles jalonan al escogimiento de un número de sujetos que puedan aportar información útil para un estudio en profundidad. Dentro de esta modalidad se eligió un muestreo de casos extremos (Rojas, 2010) al estar representados los sujetos por diferentes roles dentro del proceso social de la migración. Asimismo, se establecieron criterios de selección de los informantes claves discriminados así: a) Para los Migrantes: ser trabajador informal, migrantes temporal o de paso y trabajador formal; b) Para las entidades que atienden la población migrante: estar vinculados a los procesos sociales y conocer muy bien los procesos migratorios de la zona de frontera por un período mayor de 2 años (tabla 1).

\section{Tabla 1: Muestra intencional de la investigación}

\begin{tabular}{c|c}
\hline MIGRANTES & MIGRANTES \\
Migrante 1(M1) & Trabajador informal \\
Migrante 2(M2) & Migrante temporal (de paso) \\
Migrante 3(M3) & Empleado \\
\hline
\end{tabular}




\begin{tabular}{c|c} 
ENTIDADES ESPECIALIZADAS & CRITERIOS \\
Entidad 1(E1) & Entidad 2(E2) \\
EXPERTOS EN TEMAS MIGRATORIOS \\
$\begin{array}{c}\text { Experto 1 (EP 1) } \\
\text { Experto 2 (EP 2) }\end{array}$ & $\begin{array}{c}\text { Tener mínimo dos años de experiencia } \\
\text { en atención a la población migrante }\end{array}$ \\
\hline
\end{tabular}

Fuente: Elaboración propia (2019)

La recolección de la información de los actores migrantes fue a través de entrevistas a profundidad, la observación in situ donde ocurre la migración y con los actores sociales como una actividad consciente y planeada para indagar y obtener datos sobre hechos, conocimientos, opiniones, juicios y motivaciones (García, 2005) en la cual se interactuó cara a cara con el informante clave, en un diálogo horizontal, dialéctico y orientado por un guión de preguntas abiertas; o menos abiertas cuando se intenta verificar la comprensión de la expresión del colaborador (Moreno, 2009). Este instrumento aplicado en diferentes sesiones de tiempo a todos los actores a seleccionar (migrantes, entidades encargadas de atención a los migrantes y expertos en temas de migración) y su contenido fue grabado para su posterior decodificación.

La interpretación de los hallazgos de la investigación se generó del trato analítico de los datos cualitativos, seguido de un proceso gradual y sistemático de interpretación basado en Strauss y Corbin, en la teoría fundamentada, citado por Restrepo, (2013), especialmente en lo referido a la codificación axial de los discursos y observaciones. El proceso de reducción e interpretación de los hallazgos de la investigación se desarrolló dentro de las siguientes etapas:

a) Transcripción de las entrevistas realizadas a los informantes claves.

b) Edición de las entrevistas transcritas.

c) Codificación de entrevistas y observaciones participantes.

d) Asociación de códigos en subcategorías.

e) Integración de subcategorías en categorías.

f) Construcción del informe de resultados de la investigación.

Una vez agotado el proceso de reducción de datos cualitativos y la generación de las categorías emergentes, se presenta el informe de resultados sobre la investigación. La estructura prevé un desarrollo discursivo deductivo, partiendo desde las categorías, con los correspondientes anclajes en sus respectivas subcategorías, realizando en su interior: teorizaciones, triangulación con autores y reforzamientos con microactos de habla de los informantes clave. Este proceso se realizó para cada una de las categorías derivadas de la investigación hasta derivar en las conclusiones y, finalmente, elaborar recomendaciones que sirvan para la construcción de una política pública integral que responda al problema de la falta de atención al migrante. 


\section{ANÁLISIS DE LOS RESULTADOS}

A partir de las categorías emergentes se busca aportar un discurso teórico que, a la luz de los aportes de autores que han trabajado en temas migratorios, sea un aporte en el constructo del deber ser del derecho migratorio que fortalezca una política pública integral sobre el problema migratorio en la región.

En la sistematización de la información recabada de los migrantes, entidades que los han atendido y expertos, emergieron 5 categorías, a saber: experiencia, prioridades, política pública integral, manejo internacional y roles de la comunidad internacional. Éstas se irán explicando a partir de las 15 subcategorías que la conforman con el propósito de establecer un eje conductor contextual y teórico que permita dar cuenta de sus propiedades e implicaciones en el fenómeno migratorio.

\subsection{Categoría: Experiencia}

Dentro del análisis de esta categoría emergieron 3 subcategorías, producto de los relatos de los informantes claves (IC), entidades que los atienden (E) y expertos en tema de migración (EP), que facilitan una síntesis de las percepciones e interpretaciones que se hacen de la categoría experiencia. A continuación, se presenta el desarrollo de las subcategorías emergentes.

\section{Atención recibida}

M2: "Mis expectativas no se han cumplido y seguramente yo creo que a muchos migrantes tampoco; las ayudas son muy pocas y somos muchos los que las necesitamos". Para los migrantes ha sido difícil la llegada a un país desconocido, sintiendo en algunos casos rechazo por su condición. M3: "Me han recibido mal me han cerrado las puestas"; situación reflejada a la hora de buscar algún beneficio como lo es en salud, en donde la prioridad son los nacionales. M1: "He recibido solo la ayuda de la ONU, \$ 126.000 pesos en mercado que almacén Éxito mensualmente nos da (...) estoy en Colombia porque la crisis venezolana es muy dura, allí tengo familia y la tengo que ayudar de alguna manera”. Dentro de las expectativas de los migrantes al llegar a territorio colombiano está la de trabajar o recibir algún beneficio que les permita ayudar a sus familias en Venezuela, pero ante la dificultad de conseguir algún empleo, buscan continuar su recorrido hacia el interior del país o a países de la región como Ecuador Perú y Chile, como se evidencia en el estudio del Banco Internacional de Reconstrucción y Fomento/Banco Mundial (2018), en el que se resaltan las estadísticas de Migración Colombia, durante los primeros 9 meses de 2018, unos 724.036 venezolanos habrían hecho tránsito por Colombia hacia otro país.

La familia, así como los vínculos que se establecen alrededor de ella son factores determinantes de las decisiones de emigrar, ya que la emigración es una estrategia en la obtención de ingresos y poder garantizarles una mejor calidad de vida, bienestar y prosperidad, entre estos enfoques de la estrategia familiar se encuentran los realizados por Sandell, Sandell, Sorroza y Olivié (2007). 


\section{Trato recibido}

M1: "A pesar de todo un alto porcentaje de colombianos nos han abierto las puertas; son receptivos”. Es de resaltar que los migrantes, sienten que la población fronteriza los ha acogido bien, a pesar de que algunos se sienten excluidos por el solo hecho de ser extranjeros; lo que sugiere que se debe trabajar a nivel de educación en generar consciencia de lo que es ser migrante y no permitir los brotes de xenofobia. M2: "Que el pueblo no tome represalias. Porque la mayoría de venezolanos viene en busca de trabajo, el $90 \%$ viene a trabajar, pero hay un $10 \%$ que solo viene a causar problemas". A propósito, Julián (1981 y 1989), advierte que la inmigración no debe prohibirse bajo ningún argumento, que es como prohibir el ingreso de mercancías a un país bajo la disculpa de que afecta a los productores nacionales, que si bien puede ocurrir un efecto nocivo, en el corto plazo será transitorio, ya que en el largo plazo habrá beneficios superiores; caso contrario, como lo advierte De La Garza (2011), "la xenofobia, entendida ésta como el rechazo expresado a través de prejuicios en contra de todo aquél extranjero, teniendo en cuenta que los prejuicios son convicciones sin fundamento, con desconocimiento de los hechos, que desencadenan fácilmente la discriminación”. La xenofobia frente a los venezolanos se profundiza, como lo indica Cortés (2018), como uno de los métodos de deshumanización discursiva que los periodistas usan en nuestro contexto, sobre cómo los reporteros crean discursivamente las identidades de los migrantes y sobre cómo los noticieros construyen su aparente autoridad para representarlos. A lo anterior el uso irresponsable de las redes sociales ha generado en la comunidad fronteriza de Cúcuta, un aumento de comportamientos xenofóbicos hacia el migrante venezolano.

\section{Requerimientos}

M1: “Trabajo, ayuda, atención entre otras cosas”. M3: “Alojamiento y fuentes de trabajo principalmente el migrante llega y no tiene dónde quedarse, dónde alojarse y no puede vivir en la calle".

Para los migrantes es importante que la ayuda humanitaria del estado sea eficiente y que se faciliten procesos de regularización para poder acceder a ayudas y sobre todo fuentes de trabajo y educación. M2: "Homologación de títulos y procesos de regularización más flexibles”. Los cuellos de botella en los procesos de homologación de títulos por parte del Ministerio de Educación en Colombia son difíciles, lo que impide al migrante participar o laborar en condiciones más justas.

Para Arango (1985), la migración obedece a unas razones, dentro de las cuales se destaca el interés por irse a un país por razones de conveniencia; el origen de la migración internacional es de carácter económico y político; que es el caso de los migrantes venezolanos, quienes huyen del régimen de su país, o simplemente migran por razones económicas hacia Colombia. En estos procesos migratorios sobre todo en el caso de los profesionales, la movilidad se ha descrito como un elemento clave del hábito académico y una norma bien establecida en la vida científica, las personas que se desplazan dentro del mundo académico han sido consideradas en general como "migrantes de conocimientos" y "migrantes de talentos", talentos que han sido relegados 
por la no facilidad de condiciones de trabajo, los bajos salarios y la falta de una competencia justa por las posiciones, todo lo cual afecta negativamente a las perspectivas de empleo (Mendoza, Staniscia y Ortiz, 2020).

\subsection{Categoría: Prioridades}

En esta categoría emergen tres (3) subcategorías en los relatos de los informantes clave (IC) que analizaremos a continuación.

\section{Dificultades encontradas}

M3: "Generar dinero en Colombia es muy difícil, las fuentes de trabajo son limitadas y llegar a pedir es indignante (...) no queremos ser una carga para el estado colombiano". Competir con la mano de obra o en lo laboral en zona de frontera es difícil para el migrante venezolano ya que no tienen las mismas competencias que los nacionales, lo que les hace difícil acceder al mundo laboral; resalta D'Souza (2019), que muchos migrantes son trabajadores poco calificados del sector no estructurado, en el que los requisitos para una contratación completa son severos; sumado a la tramitología en los procesos de regularización. Para el migrante, la opción de pedir dinero es humillante, razón que ha llevado a algunos a la ilegalidad como opción y en el caso de las mujeres a la prostitución como un medio de subsistencia. En este sentido, Munk (2006) afirma que cada vez más mujeres migrantes de todo el mundo buscan trabajo lejos de casa y la prostitución es una opción frente a la dificultad de tener acceso al mundo laboral de forma legal.

\section{Necesidades sentidas}

M3: "Nadie da información sobre las ayudas o quien informe sobre los días de atención". Una de las dificultades que tienen los migrantes para acceder a las ayudas, es que desconocen las rutas de atención, que en muchos casos no son claras ni para el migrante o funcionarios de las entidades que prestan los servicios.

M2: "Que la migración se transforma en una migración productiva (...) que con la ayuda internacional que recibe Colombia en combinación con el gobierno nacional departamental y municipal y la empresa privada pudieran capacitar a los migrantes como mano de obra especializada”. En éste tema hay consenso entre los migrantes, entidades y especialistas en temas de migración, y es la necesidad de introducirlos en los procesos laborales mediante un capital semilla que soporte los emprendimientos, que capitalice el valor agregado del conocimiento; en Ngota, Rajkaran, y Mang'unyi, (2019), se evidencia que los migrantes pueden ser un factor estimulante en desarrollo de las aptitudes locales, el desarrollo y la transferencia de tecnología, las nuevas oportunidades empresariales y mejorar la inclusión en la economía globalizada. En las apreciaciones de García (2020), la migración de trabajadores con talento en todo el mundo y fuga de cerebros en Venezuela, en especial a Colombia, representa una oportunidad de crecimiento económico e innovación en los procesos productivos no solo regionales sino nacionales. 


\section{Limitaciones}

E1: "Un paquete integral de atención a las mujeres con enfermedades crónicas, mujeres lactantes y violencia intrafamiliar todo el aspecto psicosocial”. Una limitante es la poca capacidad de atención integral sobre todo a la población más vulnerable como lo son los ancianos, niños, mujeres y etnias como los Yukpas. El tema de los migrantes venezolanos desbordó la capacidad de atención esto quiere decir que E2: "el flujo que llega diario de migrantes y el acumulado presenta una tasa muy alta lo que hace que la institucionalidad no pueda darle respuesta”.

Se puede afirmar según los relatos de los informantes claves que, el alto nivel de flujo migratorio ha desvelado las grandes limitaciones que en tema de migración se presentan, una es la inexperiencia del estado colombiano frente a la magnitud del problema. Otro elemento ha sido la politización de la migración ya que se ha convertido en tema de campañas políticas a nivel regional, nacional y aún internacional como tema geopolítico. El cierre de fronteras de algunos países como Ecuador y Perú, han generado un embotellamiento de la población migrante, lo que ha dejado solo al estado colombiano con el problema, y si a esto se le suman las relaciones binacionales rotas, la situación empeora.

Como lo manifestó E1: "en cuanto a Cooperación Internacional también hay un desgaste presupuestal”. En este sentido existe un agotamiento de lo presupuestal no solo a nivel nacional, sino en las ayudas internacionales adicional a las limitaciones del recurso humano para atender a los grandes flujos de población, agravando más el fenómeno migratorio.

\subsection{Categoría: Política pública integral}

Dentro del análisis de esta categoría emergen 3 subcategorías en los relatos de los informantes clave (IC) que serán las instituciones que atienden a los migrantes (E) y los expertos en temas de migración (EP).

\section{Fenómeno migratorio}

Para los especialistas en temas migratorios consultados el tema migratorio puede mirar de dos maneras: EP1: "una donde se ve la migración como algo negativo que termina afectando los mercados laborales (...) en Colombia nunca hubo política de migración cuando comenzó la migración”.

Lo anterior sugiere la necesidad de asumir como enfoque epistemológico la construcción de un nuevo perfil del migrante. Los expertos (EP) enfatizaron en la necesidad de construir una política integral sobre migración, la cual no existía en Colombia, ésta se inicia con los procesos migratorios, pero de una forma muy incipiente; ahora lo que hay que entender en materia de política pública, es lo relacionado con la acción sobre el sujeto; la defensa de los derechos para el que se va, o para el que llega; en este caso los venezolanos. 
Desde la academia diferentes actores están planteando una política que garantice los derechos a no migrar porque la migración se ha vuelto un proceso de desplazamiento forzado; para EP2: "el derecho que mi país me garantice que yo me pueda quedar y tenga una realización personal y profesional, que mis hijos pueden tener libre desarrollo; una política debería incluir ese derecho fundamental a preservar que ese individuo, si se quiere quedar en su país, se quede con todas las garantías".

Otro enfoque es comprender la esencia del migrante desde una perspectiva positiva donde se observa a la migración como una oportunidad desde el derecho, entendiéndola como una necesidad humana; una visión antropológica en donde la misma historia del homo sapiens ha sido un proceso de evolución y de migración. EP2: "Desde el punto de vista religioso también se puede ver como el pueblo de Israel permaneció en una diáspora continua; desde la antropología física se encuentra el caso del Éxodo en el Antiguo Testamento con Moisés, en el Evangelio de Lucas, en el Nuevo Testamento, cómo Jesús fue un migrante; es entender que la migración ha formado parte de la naturaleza humana”.

En el caso de EP1: "entender que no podemos invocar esos nacionalismos, esas barreras que unas culturas han construido es un reconocer al otro como ser humano en un sentido real porque hay un marco jurídico a nivel Internacional que Colombia debe reconocer esa categoría de migrantes”, fenómeno que se da por algún fenómeno de crisis económica catástrofe natural variación del cambio climático guerras, son situaciones que se dan y deben ser protegidas desde una perspectiva del derecho, desde lo ancestral. Resaltan Losoncz y Marlowe (2020), los migrantes se enfrentan a una serie de barreras y amenazas sistémicas de las instituciones gubernamentales relacionadas con el cultivo de la identidad propia.

Un segundo aspecto positivo es observado desde una perspectiva económica como una inversión, no como gasto; EP2: "en el caso de Colombia hay una política económica que parte de la idea de que la migración es un gasto o una carga política; esta visión hay que replantearla ya que puede ser una oportunidad de relevo generacional”. Según datos del Censo Nacional de Población y Vivienda (2018), en Colombia se evidencia la transformación que esa población ha tenido en esta década y media reciente, por ejemplo, la franja de edades entre 15 y 65 años es de $68.2 \%$ lo que indica que la población colombiana es en un grado significativo una población envejecida. Hoy, por cada 100 personas menores de 15 años hay 40,4 adultos mayores de 65, mientras que, en el Censo Nacional de Población y Vivienda (2005), esa proporción era de 20,5 adultos, es decir, se ha duplicado el número de personas de ese rango de edad, frente a la población más joven.

Es aquí cuando vemos a los migrantes venezolanos, en su mayoría jóvenes mayores de 18 años son una oportunidad de renovación precisamente de esa sociedad, de esa pirámide social y una forma de refrescar la cultura; los rangos de la migración está entre los 18 y 35 años, en ese sentido favorece ese aspecto, ya que es una población económicamente activa y productiva; es ahí donde se abre la puerta a una política pública integral; ver esa oportunidad de transición demográfica e incorporarla a los procesos económicos y sociales. 
Por lo anterior, se aborda el estudio "Monitoreo de flujo de la migración venezolana en el Perú”, elaborado por la Organización Internacional para las Migraciones (OIM, 2019), en el cual los resultados obtenidos señalan que la mayoría de migrantes venezolanos que ingresaron al Perú en los últimos meses son jóvenes en edad productiva (entre los 18 y 39 años), que además cuentan con estudios secundarios o superiores completos. Aquellos que cursaron una carrera universitaria lo hicieron en áreas de administración, ciencias, ingeniería y educación.

Es de resaltar que los informantes claves sobre todo los especialistas en temas migratorios "EP1 y EP2 enfatizan sobre la idea de abordar el tema migratorio a partir de dos perspectivas: una donde se ve la migración como algo negativo que termina afectando los mercados laborales y termina desplazando la fuerza laboral colombiana, sustituyéndola por fuerza laboral venezolana y bajando la tasa de salarios, por lo cual, los nacionales los ven como una amenaza generando problemas de inseguridad y el aumento de la xenofobia”. Estas situaciones de rechazo a los migrantes como algunos han pensado, han provocado el incremento de los delitos donde efectivamente grupos vulnerables colombianos luchan por acceder a los servicios básicos y entonces encuentran en la migración o en los venezolanos una competencia desleal. Así, estos dos grupos vulnerables, entran en una puja por conseguir esos recursos, los cuales, en ciudades como Cúcuta, son limitados.

En el caso de EP1: "hay que desmontar esa visión pesimista que lleva a posturas xenofóbicas creadas y equivocadas”, a lo que Cortina (2017), refiriéndose al caso de España, señala que en donde se presenta odio a los pobres o aporofobia, no nos gusta, son los pobres; los migrantes ricos sí, ellos dejan recursos no vienen a presentar dificultades ni mendigar, y donde la respuesta a este drama migratorio se debe dar desde nuestra condición humana con altura y es hospitalidad cosmopolita, entendida como virtud moral y deber jurídico.

En palabras de EP2: "es una versión perversa, representamos al otro con una imagen negativa. Venimos con una tradición de violencia muy cercana del país y entonces tenemos una estética de la violencia y de la pobreza muy marcada y encontramos percepciones como que hay que votar por candidatos que fomenten la limpieza de los pobres porque esos estéticamente no se ven bien”. Hay que recordar que antes de la migración venezolana, Cúcuta tenía una tasa de desempleo alta, ocupando, según el Departamento Administrativo Nacional de Estadística (DANE), en el mes de junio de 2019, el cuarto lugar a nivel nacional con el 15,6\%. En comparación al mismo lapso del año anterior, la tasa de informalidad que tiene la ciudad es igual básicamente a la que tenía el fenómeno migratorio con los venezolanos; el problema no son los migrantes, es un modelo de desarrollo que la migración lo refuerza, pero que viene funcionando paupérrimamente (tabla 2).

\section{Tabla 2: Población ocupada, desocupada e inactiva}

Cúcuta A.M ${ }^{*}$

\begin{tabular}{l|l|l|l|l}
\hline \multirow{2}{*}{ Poblaciones } & \multicolumn{4}{|c}{ Variación } \\
\cline { 2 - 5 } & Nov 18 - Ene 19 & Nov 19 - Ene 20 & noviembre - enero (2018-2020) Absoluta & $\%$ \\
\hline
\end{tabular}

Áreas metropolitanas. 


\begin{tabular}{c|c|c|c|c}
\hline Ocupados & 331 & 344 & 13 & 3.9 \\
Desocupados & 67 & 67 & -1 & -0.9 \\
Inactivos & 276 & 272 & -3 & -1.2 \\
\hline
\end{tabular}

Fuente: DANE, GEIH, ${ }^{5}(2020)$

Un aspecto que se debe fortalecer es la experiencia o valor agregado que han desarrollado los colombianos de frontera sobre todo los nortesantandereanos, en procesos de migración externa e interna; como víctimas del conflicto son resilentes y entienden a los migrantes que arriban. Perciben al migrante una oportunidad de fortalecer los procesos productivos encaminados al desarrollo industrial, empresarial, los saberes y experiencias para fortalecer la investigación, sobre todo en la academia.

Tipayalai (2020), considera que el inmigrante altamente cualificado tiene un impacto positivo en el crecimiento económico regional en el caso de Tailandia, (...) por lo tanto debería centrarse más en traer trabajadores extranjeros altamente cualificados ya que probablemente promueva la innovación y la tecnología nuevas y atraiga más inversión extranjera, aumentando la transferencia de conocimientos y mejorando la productividad laboral en general; ese reconocimiento del migrante en la sociedad beneficia la productividad, busca la eliminación de los límites fronterizos entre naciones, reivindica el comercio y la competencia, y ve importantes aciertos sobre el crecimiento poblacional.

Aquí es pertinente resaltar a Bak-Klimek, Karatzias, Elliott, Dickson, A.d, MacLean (2020), quienes ven que la migración no es un simple acontecimiento estresante en la vida, sino que puede tener un efecto positivo en muchos aspectos de la vida de los inmigrantes y puede ofrecer oportunidades de crecimiento personal.

\section{Aspectos por mejorar}

EP2: "El documento CONPES que trae ocho elementos que forman parte de la política migratoria tiene dos grandes falencias: una es la concepción de temporalidad, una política pública que es concebida como fenómeno a corto plazo mediático y no a largo plazo y no se puede afirmar que sea un documento de política pública”.

El CONPES 3950 de 2018, no es un documento de política pública, sino que se considera un documento base para la construcción de una política pública, en él se generan algunas líneas estratégicas, elementos que indican cómo fortalecer la registradora, salud, educación etcétera; no es específico y carece de presupuesto y de la robustez institucional, esto quiere decir que para poder atender la migración en Colombia hay que crear toda una institucionalidad así como se creó para atender el desplazamiento forzado (Unidad de Reparación de las Víctimas).

Las instituciones de salud como el Hospital Universitario Erasmo Meoz, es el centro de salud más importante de Cúcuta y del departamento Norte de Santander con categoría nivel cuarto de complejidad, en el que se atiende la población venezolana que no cuenta con el recurso humano, físico ni económico. EP2: "Es de resaltar que

${ }^{5}$ Gran encuesta integrada de hogares (GEIH) Mercado laboral - Históricos. 
el Gobierno Colombiano ha realizado cosas importantes y concretas en temas como educación, salud, de registro el tema de trabajo, como la tarjeta de movilidad fronteriza (TMF); cosas muy puntuales, pero no la política concreta, (EP2)”.

Es de vital importancia como lo manifiesta EP1: "el engranaje entre las instituciones públicas, privadas y las de cooperación internacional para poder lograr lo que propone el CONPES"; que las ayudas de los entes nacionales e internacionales algún porcentaje se destine como capital semilla o a proyectos productivos con el apoyo de la empresa privada para el dinamismo de las economías locales.

Los especialistas en temas de migración EP1 y EP2 coincidieron que: "los procesos de regularización son verdaderos cuellos de botella; en todos los aspectos son poco flexibles". Frente a esta limitante, a los migrantes se les dificulta acceder no solo a los beneficios otorgados, sino al mundo laboral más cualificado. Sin una regularización real y flexible, los problemas de los migrantes tenderán más a agudizarse que a tener una solución real. En este aspecto se presenta aquí un reto para el derecho, en cuanto se debe trabajar en una legislación más flexible que pueda aliviar la situación del migrante.

\section{Elementos para incorporar}

EP1: "Una política pública necesaria pasaría primero desde lo que ha sido la migración venezolana por un trabajo de concienciación social”. Este sería el nuevo reto de una política que tendería a desarrollarse bajo una metodología considerando la participación de todos los actores interesados en la solución del problema, tales como las organizaciones de la sociedad civil y académicos; ejercicio que debe incorporar a los actores que están más "afuera", es decir insertados en la realidad migrante. Esto significa que una política tendría que ser como lo manifestó EP2: "lo primero es actuar con la ciudadanía, construir ciudadanía, enseñar que somos, desde política educativa, una sociedad multicultural". Necesitamos una política de construcción de convivencia de adaptación y eso lo hacen desde la escuela, allí se debe enseñar a aceptar al otro, esto reduciría la agresión; tarea que Rachmadtullah y Rasmitadila (2020) explican, y no es otra que la implementación del multiculturalismo en la educación, una actitud de tolerancia hacia los demás causará un sentimiento de afecto y también un fuerte sentido de la hermandad, tarea que facilitaría la convivencia con el migrante.

Se debe partir de un concepto de ciudadanía multicultural, en la que la presencia del otro, en este caso el migrante, aporta a los diferentes procesos sociales. EP2: "son perspectivas visiones del mundo como fue el caso de los americanos para la sociedad americana lo que la nutre independientemente de la nacionalidad; Latinos Norteamericanos, Italianos, Griegos o Alemanes y en esas construcciones cotidianas de cultura eso es lo que le da importancia nosotros no hemos logrado entender eso porque de alguna manera somos muy muy locales entonces aquí un tema de política es tener visiones y generar políticas globalmente que permitan la construcción de civilización”. Aquí los países deben trabajar en la construcción de nuevas leyes que tengan en cuenta los fenómenos migratorios. 
Fortalecer pedagógicamente la atención a los migrantes desde lo institucional, EP1: "dar una mejor atención, parte del respeto al sujeto objeto de la migración, no dando un servicio de mala calidad; cualquier ayuda si no valoramos al otro es una relación desigual”, sólo se construyen relaciones fuertes cuando observamos al migrante en una condición similar a la mía, solo así podemos hablar de respeto y solidaridad; las políticas solidarias consisten en no discriminar, involucrar a todos los que necesitan beneficios, implica un proceso de construcción de convivencia con el otro, es observar a los migrantes como sujetos de derecho, y que no pueden ser estigmatizados bajo una sola categoría. Según Baker (2020), los refugiados y migrantes no pueden ser representados como víctimas sin ningún tipo de agencia, son discutidos en términos jurídicos que sirven para deshumanizarlos, y son repetidamente "cuantificados" como una categoría homogénea y potencialmente problemática.

E1: "Es prioritario crear una política pública de manera sectorial concreta en temas de salud, educación, generación de ingresos y en procesos de regularización, esto tiene que ver con creación de una institucionalidad a la que se le pueda delegar funciones y tenga la capacidad de dar respuesta”. En ese sentido, que se asigne un rubro que quede incorporado no solo en lo nacional, sino también en lo local; se necesita que los gobernantes, sea cual sea el nivel de gobierno, alcaldes o gobernadores, dejen en sus planes de gobierno, en sus líneas estratégicas la asignación de rubros para atender la migración y que esas líneas estratégicas se conviertan posteriormente en una política pública fortalecida con la participación de todos los actores implicados.

Para E2: “Una política pública debe contener financiación probable o real. En el caso de los documentos CONPES, éstos se han quedado en buenos documentos estratégicos pero las realizaciones de sus planes no se materializan por falta de recursos"; la financiación es fundamental para poder impulsar realmente una política pública que incluya el acceso a todos los derechos y que logre que todos los migrantes puedan vincularse a la sociedad colombiana y lograr una producción que coadyuve su mantenimiento y también la economía del país. Es vital como decía EP1: "Quitarnos el asistencialismo ya después de estos dos años de crisis de fuerte movimiento migrantes, se empieza a fortalecer ciertos comportamientos como el recibir ayudas"; costumbres que se tienen que erradicar porque el migrante no empieza a producir, situación que se ve como una debilidad del gobierno colombiano, reto que se debe asumir para ir cambiando el paradigma del asistencialismo.

\subsection{Categoría: Manejo internacional}

Dentro del análisis de esta categoría emergen cuatro (4) subcategorías en los relatos de los informantes clave (IC).

\section{Apoyo internacional}

EP1: "En el caso colombiano ha sido la comunidad internacional un gran apoyo, sobre todo en la transferencia de conocimientos", se fortalecieron en la administración pública capacidades en ese aspecto. Las ayudas internacionales han sido un alivio, 
pero en el caso colombiano por el alto volumen migratorio, el más grande visto en el mundo posmoderno, ha agotado los recursos; sumado a lo anterior, las posiciones radicales de países vecinos como cerrar fronteras, generando un hacinamiento de venezolanos en todo el territorio, ha agudizado más los problemas existentes en el país.

Los países de la región como Ecuador y Perú han sido muy hostiles; éstos dos gobiernos exigirán pasaporte a venezolanos como requisito para ingresar, en medio de un creciente flujo migratorio; situación que ha agravado problemas de violación de los derechos humanos de los migrantes, y una política pública debe hacerse desde los consensos regionales para que sea más efectiva. EP2: "Si no hay solidaridad de los países de la región será muy difícil asumir una crisis de esta magnitud en el país”. Es importante que exista esa solidaridad regional en estos temas ya que ningún país está exento de problemas de esa naturaleza.

Como los gobiernos son cambiantes y las instituciones cambian, E2 afirma que: "atender seres humanos es directamente proporcional a recursos, lo que ha limitado brindar una mayor cobertura en salud, educación, acceso al trabajo y protección a los menores, dificultando el control migratorio". Es importante recordar que Colombia es un país con recursos limitados, que trabaja bajo un esquema de planeación que no contempla la población migrante, pues difícilmente las consecuencias que estas provocan son previstas y, por lo tanto incluidas en los presupuestos y en la planeación para el desarrollo de los estados.

\section{Cooperación internacional}

EP1: "La cooperación internacional se ha presentado como una oportunidad de negocio o de alguna manera de lucro para quienes asumen roles dentro de este proceso”; el manejo de dineros ha fortalecido agencias, fundaciones y ONG's; y alrededor de ellas todos los procesos que de alguna manera cumplen un rol dentro de los flujos migratorios respecto a la atención del migrante; es una oportunidad laboral para muchas personas, que de esta manera pueden beneficiarse del problema.

\section{Migración}

Hay que reformar aquellas leyes que atienden el problema, tal como se ha hecho ya en otros países y no eliminarlas en cada transición de gobierno que se presenta; en ese sentido la migración se ha convertido también en una moneda política que el gobierno colombiano utiliza, siendo flexible con los venezolanos para generar una imagen positiva ante la comunidad internacional en esa lucha contra el régimen de Maduro.

\section{Flexibilidad}

Colombia ha asumido una actitud muy flexible frente a los migrantes, EP2: "Colombia ha sido relativamente generoso, aunque está muy lejos de los parámetros internacionales”, y en el sentido de que muchos derechos están establecidos, en la práctica así sea regularizado o no, ha ido creando una legislación que busca favorecer a los migran- 
tes; en el caso de los permisos temporales, Colombia no ha cerrado las fronteras a los migrantes, facilitando el tránsito hacia el interior del país y posteriormente hacia países vecinos como Ecuador, Perú y Chile. A partir del incremento de la migración de venezolanos hacia Colombia, se ha identificado que muchos de estos migrantes tienen como principal actividad la delincuencia y la violación constante al marco normativo de Colombia, no obstante, los procesos de deportación han sido casi nulos. EP2: "Ha facilitado lo laboral y la informalidad en la mayoría de los casos, situación que ha agudizado la crisis económica ya existente”.

\subsection{Categoría: Roles comunidad internacional}

Dentro del análisis de esta categoría emergen dos (2) subcategorías en los relatos de los informantes clave (IC) que serán los expertos en temas de migración (EP).

\section{Instrumentalización}

EP1: "Yo creo que sí creo que sea instrumentalizado mucho, hay migraciones que nos duelen más que otras”; algunas han generado cercos humanos, como las migraciones haitianas de las cuales nunca se dijo nada, algunas migraciones han sido objeto de selección mural de posiciones internacionales hipócritas y eso ha pasado con la migración venezolana una relación de poder geopolítico a nivel internacional.

Se han hecho cosas positivas en temas migratorios, pero la dificultad es el comportamiento de algunos migrantes venezolanos, logrando que algunos países de la región endurecieran sus posiciones como fue el caso de Ecuador y Perú cerrando sus fronteras; situación que ha generado un represamiento de población migrante en territorio colombiano, lo que ha agudizado mucho más el problema de ese país; incluso se han agudizado problemas que ya existían antes del fenómeno migratorio. No obstante, se debe reconocer que Colombia, a pesar de las limitantes que ha tenido, ha recibido ayuda de agencias internacionales de manera significativa.

\section{Poder geopolítico}

EP2: "Creo que éste fenómeno migratorio al igual que otros en términos generales no ha tenido un buen manejo”, EP1: "basta ver lo que está ocurriendo en Europa con la migración desde el Norte de África lo que está ocurriendo en Centroamérica y Estados Unidos con el grupo centroamericano con la política de Trump, hay más intereses geopolíticos".

Se pudo observar cómo algunos países latinoamericanos comenzaron a crear algunas normas y legislaciones positivas, pero otros le han cerrado sus puertas al migrante violando un derecho internacional humanitario como es el libre tránsito y el derecho para migrar.

EP2 afirma que: "Latinoamérica está muy lejos de los parámetros internacionales en el sentido de que muchos derechos están establecidos, pero en la práctica, así sea regularizado o no, se deben tener en cuenta”. Esto se puede evidenciar en los permi- 
sos temporales que se dan por lotes y que posteriormente cambiaron las condiciones; se debe trabajar a nivel de política pública migratoria en los procesos de regularización, EP1:"un inmigrante no está regularizado, no tiene derechos, los tiene de manera mínima y circunstancial, más cuando ni siquiera tiene un documento identidad; por ahí es complicada la cosa entonces yo diría que de conjunto en el mundo Latinoamérica se ha echado para atrás el término de manejo en migratorios”.

\section{LAS TAREAS PENDIENTES}

Una de las encomiendas importantes siendo producto de la investigación, es la sanción de una ley migratoria que implemente los recursos destinados a la construcción de políticas públicas dirigidas a la atención de migrantes con contenido integral. Los estados, como el nuestro, a partir de la experiencia vivida con la migración masiva de venezolanos a territorio colombiano, ya deben pensar en sancionar una ley más integral que le permita direccionar rubros para la atención migrante.

Otra de las recomendaciones está orientada a la implementación de una política pública de inclusión que vaya desmontando el asistencialismo al migrante y con ello, se logre un proceso donde se le permita incorporarse a la vida productiva del país y con ello se reduzcan los sentimientos de xenofobia. Es importante una política pública de atención al migrante contemplando una mayor flexibilización en los procesos de regularización, que le permita al migrante una mayor vinculación al campo laboral y no fortalecer un asistencialismo que en nada le beneficia a él ni a los gobiernos que los reciben. Los procesos migratorios hay que asumirlos con otra óptica más holística, que facilite procesos de integración social menos dolorosos.

Se deben de fortalecer los puestos de atención en frontera, ya que las instituciones están desgastadas y dada la gran cantidad de migrantes, necesitan fortalecer su estructura organizacional para el control y la atención de los extranjeros, así como hacer más eficientes sus procesos. El gobierno nacional debe contemplar en sus planes presupuestales los recursos que garanticen la repotencialización de las instituciones encargadas de la atención de población migrante, formando recurso humano suficiente para atender este tipo de fenómenos como el presentado con la migración venezolana.

La política pública migratoria debe tener objetivos claros y con metas a corto, mediano y largo plazo encaminadas al aprovechamiento de la fuerza laboral de los migrantes para el desarrollo del país. Es decir que los estados asignen rubros para fortalecer emprendimientos y lograr fortalecer a los gobiernos locales como alcaldías y gobernaciones, en unas líneas estratégicas que les permitan implementar mecanismos de atención a la población migrante.

Una política pública integral debe partir como su nombre lo indica de un proceso de integración de todos los sectores involucrados en los procesos migrato- 
rios: migrantes, ONG's, organismos gubernamentales locales, nacionales e internacionales. La construcción debe hacerse con todos los actores locales, como lo son las alcaldías y las gobernaciones, para que se puedan generar líneas de atención al migrante y cuenten con el presupuesto asignado para su desarrollo.

Uno de los actores a los cuales se les debe de considerar su participación es la academia como organismo dinámico en los procesos de investigación y construcción de estrategias pedagógicas encaminadas a fortalecer las relaciones amigables con el migrante; es cambiar el paradigma negativo de la migración y presentarla como una oportunidad de crecimiento en los diferentes aspectos como lo económico, cultural y social; procesos que favorecen la interculturalidad.

Asimismo, es perentorio fortalecer los tratados internacionales y regionales, en los cuales los gobiernos garanticen la cooperación conjunta y el respeto por los derechos humanos del migrante, derechos que son vulnerados en el caso de este fenómeno como el venezolano, en el que algunos países de la región endurecieron sus posiciones y cerraron sus fronteras al migrante.

Finalmente, una política pública debe partir de una caracterización de la población residente en Colombia, ejercicio que no ha sido realizado y es clave para dirigir las políticas migratorias en cuanto a trabajo y educación a los migrantes y también para el proceso de inclusión a la economía.

\section{REFLEXIONES FINALES}

Un primer elemento que se logró identificar es: la percepción de los migrantes al llegar a territorio colombiano, llegar a un país desconocido genera una serie de incertidumbre y angustia frente a lo que puede encontrar, no solo lo ajeno del territorio; se encuentra con otras costumbres y normatividad existente: la condición de extranjero sumado al desarraigo familiar y al hecho de haber dejado a su familia en una condición difícil aumenta más la angustia existencial.

Para el migrante es muy difícil obtener dinero para su subsistencia, llegar a mendigar no solo dinero, sino conseguir lo básico como vivienda y alimentación es una lucha, que se agudiza por desconocimiento de las rutas de atención, sino por los mismos problemas de regularización y de competencia desleal no solo entre sí, también con los habitantes de frontera, producto del alto volumen de población migrante.

Son preocupantes los cuellos de botella en los procesos de regularización y de homologación de los títulos que les permita acceder al mundo laboral y no ser una carga para el estado, en este caso el colombiano. Estos procesos difíciles hacen que el migrante viva una situación de desigualdad; los procesos de inclusión son casi nulos, lo que ahonda más la situación de forastero y facilita los brotes de xenofobia. 
Las rutas de atención para el migrante no son claras y, en su mayoría, las entidades del estado colombiano no saben cómo actuar frente a un migrante. No se conocen los protocolos de atención y esto genera un clima de exclusión; en este sentido, los privilegios son en primer lugar para los nacionales, y a los migrantes, en algunos casos, se les vulneran los derechos fundamentales, propiciando una violación fragante al Derecho Internacional Humanitario (DIH), rama del derecho internacional destinado a limitar y evitar el sufrimiento humano en tiempo de conflicto, como es el caso del venezolano.

Otro aspecto para resaltar es el desgaste no solo institucional y humano, sino presupuestal de las instituciones nacionales e internacionales encargadas de la atención al migrante, como consecuencia del alto volumen de migrantes presentado en esta zona de frontera, lo que ha desbordado la capacidad de atención, no solo del gobierno colombiano, sino de los organismos internacionales.

Por tanto, se necesita un mayor compromiso de los países de la región para enfrentar fenómenos tan grandes como el venezolano y no se pueden asumir medidas como el cierre de fronteras dejando a un solo país con el problema. Se debe partir de que el migrante es sujeto de derecho y que se le deben de garantizar los derechos humanos; derechos que deben ser asumidos con un mayor compromiso y veeduría internacional o de organismos como la Organización de las Naciones Unidas (ONU) y la Organización de Estados Americanos (OEA).

En el departamento Norte de Santander no hay políticas públicas orientadas a la atención al migrante, en este orden de ideas se requiere la adopción de una política que parta de la premisa de que el problema es multifactorial y por ende es detonante de una gran variedad de problemas en diversos sectores de la dinámica social de cualquier país. En este sentido y con enfoque hacia nuestra investigación, es necesaria la construcción de una política que integre una gran variedad de aspectos que atiendan en conjunto las necesidades que tienen los migrantes en ese departamento.

El diseño de la política pública de atención al migrante debe ser una iniciativa que debe estar incluida en los programas de gobierno de los alcaldes y gobernadores, recientemente electos, a fin de crear conciencia de que el fenómeno migratorio venezolano hace parte de la situación actual de la región con la cual debemos convivir y con ello eliminar los sentimientos xenofóbicos y lograr el aprovechamiento de esta población en la vida productiva del país.

Una política pública sobre migración se debe considerar desde la construcción e identificación real de problema y no desde los escritorios; se debe considerar la aplicación de una metodología donde se desarrolle un proceso de participación de todos los actores involucrados, como son los mismos migrantes, las entidades que asumen la tarea de su acompañamiento; igualmente, las mesas de trabajo en ese proceso de construcción deben involucrar los entes gubernamentales locales como lo son las alcaldías y las gobernaciones. 
Es necesario el trabajo pedagógico con las comunidades locales, reforzado con la academia sobre cómo ver al migrante desde una óptica positiva, no como una oportunidad de reforzar la internacionalización de los currículos, sino como un proceso que fortalece la interculturalidad y que aporta positivamente a los desarrollos económico, sociales y culturales de un país.

\section{REFERENCIAS}

1. Arango, J. (1985). Las "Leyes de las Migraciones" de E. G. Ravenstein, cien años después”. Revista Española de Investigaciones Sociológicas, 32, 7-26.

2. Bak-Klimek, A., Karatzias, T., Elliott, L., Dickson, A. y MacLean, R. (2020). Positive Changes and Appreciation of Life Among Economic Immigrants in Scotland. Journal of International Migration and Integration, 21(1), 57-76

3. BANCO MUNDIAL. (2018). Migración desde Venezuela a Colombia: impactos y estrategia de respuesta en el corto y mediano plazo", Washington, D.C. Disponible en: https://r4v.info/es/documents/download/66643

4. Baker, M. (2020). Rehumanizing the migrant: the translated past as a resource for refashioning the contemporary discourse of the (radical) left. Palgrave Communications, 6(1),12.

5. Censo Nacional de Población y Vivienda 2018 en Colombia. Disponible en: https://www.dane.gov.co/index.php/estadisticas-por-tema/demografia-y-poblacion/censonacional-de-poblacion-y-vivenda-2018/cuantos-somos

6. Censo Nacional de Población y Vivienda 2005 en Colombia. Disponible en: https://www.dane.gov.co/index.php/estadisticas-por-tema/demografia-y-poblacion /censo-general-2005-1/censo-general-2005

7. CONPES 3950 de 2018. Disponible en: https://colaboracion.dnp.gov.co/CDT/Conpes/Econ\%C3\%B3micos/3950.pdf

8. Cortina, A. (2017). “Aporofobia, el Rechazo al Pobre”, en Paidós, Barcelona, 196p.

9. Cortés, C. (2018). Xenofobia y periodismo: Colombia y la migración, en Universidad de La Sabana. Colombia, 21(4), 960-963

10. De La Garza, C. (2011). "Xenofobia”, Laboreal [En línea], Volume $7 \mathrm{~N}^{\circ} 2$. Disponible en: http://journals.openedition.org/laboreal/7916

11. DEPARTAMENTO ADMINISTRATIVO NACIONAL DE ESTADÍSTICA DANE 2019. Disponible en: https://www.laopinion.com.co/economia/cucuta-registro-156-de-dese mpleo-en-junio-181457\#OP 
Julio CÉSAR CARVAJAL RodRíguez / http://orcid.org/0000-0003-4517-8817. / Correo electrónico: departamentodeeducacion@cúcuta.udes.edu.co JosÉ DE Jesús NúÑEz RodRíguez / http://orcid.org/0000-0002-4120-0215. / Correo electrónico: jo.nunez@mail.udes.edu.co PAUlo CÉSAR Lugo Rincón / https://orcid.org/0000-0002-1571-9080. / Correo electrónico: paulolugo@hotmail.com

12. D’Souza, E. (2019). Migrants and Informal Casual Labour Markets. Indian Journal of Labour Economics, 62(4), 533-548.

13. DTM RONDA 5. Monitoreo de flujo de población venezolana en el Perú. Disponible en: https://reliefweb.int/sites/reliefweb.int/files/resources/DTM_MIGRACIONVENE ZUELA_R5_2019.pdf

14. Fernández, R. (2005). El cuestionario, recomendaciones metodológicas para el diseño de un cuestionario. Limusa, México, 120.

15. Fête, M., Aho, J., Benoit, M., Cloos, P. y Ridde, V. (2019). Barriers and recruitment strategies for precarious status migrants in Montreal, Canada. BMC Medical Research Methodology 19(1),683.

16. Garcia, D. (2020). Brain drain in Venezuela: the scope of the human capital crisis. Human Resource Development International, 23(2), 188-195.

17. Guillén, J., Menéndez, F. y Moreira, T. (2019). Migración: Como fenómeno social vulnerable y salvaguarda de los derechos humanos. Revista de Ciencias Sociales Revista de Ciencias Sociales 25(1), 281-294.

18. Ibolya, L. y Jay, M. (2020). Regulating Immigrant Identities: The Role of Government and Institutions in the Identity Construction of Refugees and Other Migrants. Journal of International Migration and Integration, 21(1), 117-132.

19. Julian, S. (1989). The economic consequences of inmigration. Basil Blackwell published in association with The Cato Institute, printed in the USA, 430.

20. Katikar Tipayalai. (2020). Impact of international labor migration on regional economic growth in Thailand. Journal of Economic Structures, 9(1),15.

21. Lama, B., Rajkaran, S., Mang'Unyi, E. y Mang'unyi, E. (2019). African immigrant entrepreneurs in South Africa: Exploring their economic contributions. Entrepreneurial Business and Economics Review,7(4), 33-56.

22. Martínez, M. (2009). Epistemología y Metodología Cualitativa en las ciencias sociales. Trillas, México. P. 168.

23. Mazuera, R., Albornoz, N., Cuberos, M., Vivas, M. y Morffe, M. (2020). Sociodemographic Profiles and the Causes of Regular Venezuelan Emigration. International Migration. Disponible en: https://onlinelibrary.wiley.com/doi/full/10.1111/imig.12693

24. Mendoza, C. y Staniscia, B. y Ortiz, A. (2020). "Knowledge migrants" or "economic migrants"? Patterns of academic mobility and migration from Southern Europe to Mexico. Population, Space and Place 26(2), e2282

25. Moreno, S. (2009). Descubriendo mi sabiduría corporal. Focusing, 189. 
26. Munk, V. (2006). Migration and sex work the dilemmas of illegality. Osteuropa 56(6), 255-259.

27. Rachmadtullah, R. (2020). The role of civic education teachers in implementing multicultural education in elementary school students. Universal Journal of Educational Research, 8(2), 540-546.

28. Restrepo, D. (2013). La Teoría Fundamentada como metodología para la integración del análisis procesual y estructural en la investigación de las Representaciones Sociales. Revista CES Psicología, 6(I), 122-133.

29. Rojas, B. (2010). Investigación cualitativa. Fundamentos y praxis, Fedupel, Caracas, 196.

30. Sandell, R., Sorroza, A. y Olivié, I. (2007). Inmigración: ¿un desafío con oportunidades?. Atlantic Conference, Sevilla. Disponible en: https://core.ac.uk/download/pdf /42965966.pdf

31. Strauss, A. y Corbin, J. (2002).Bases de la investigación cualitativa. Técnicas y procedimientos para desarrollar la teoría fundamentada. Editorial Universidad de Antioquia, 354 